\title{
and optimal weighted least-squares method for training tree tensor networks
}

\section{Cécile Haberstich ${ }^{1,2} \dagger$}

${ }^{1}$ LMJL (UMR CNRS 6629), Centrale Nantes

\section{High-dimensional approximation}

We consider a pair of random variables $(X, Y)$ such that $Y=u(X)$, where $X=\left(X_{1}, \ldots, X_{d}\right)$ is a random vector and $u: \mathcal{X} \rightarrow \mathbb{R}$ is a function. In the context of Uncertainty Quantification, $Y$ is the output of a numerical code and $X$ are the input parameters.

When $u$ is costly to evaluate, we replace $u$ by an approximation $u^{*}$.

\section{Objectives:}

- Construct an approximation $u^{*}$ using only a few evaluations of $u$

- Exploit low-rank structures

- Propose a sample-based algorithm with guaranteed stability

\section{Tree-Based Tensor formats}

$u$ is in $L_{\mu}^{2}$, with $\mu$ a product probability measure on $\mathcal{X}$.

$L_{\mu}^{2}$ is identified with $L_{\mu_{1}}^{2} \otimes \ldots \otimes L_{\mu_{d}}^{2}$.

For $\alpha \subset D=\{1, \ldots, d\}, u$ is identified with a bivariate function in $L_{\mu_{\alpha}}^{2} \otimes L_{\mu_{\alpha} c}^{2}$.

A function $u$ with $\alpha$-rank $r_{\alpha}$ :

$$
u\left(x_{1}, \ldots, x_{d}\right)=\sum_{i=1}^{r_{\alpha}} v_{i}^{\alpha}\left(x_{\alpha}\right) v_{i}^{\alpha^{c}}\left(x_{\alpha^{c}}\right)
$$

If a function $u$ has the above representation for all $\alpha \in T$ where $T \subset 2^{\{1, \ldots d\}}$, and $T$ is a dimension partition tree, $u$ has a representation in tree-based tensor format $\mathcal{T}_{r}^{T}$.

$$
\{1,2,3,4\}
$$

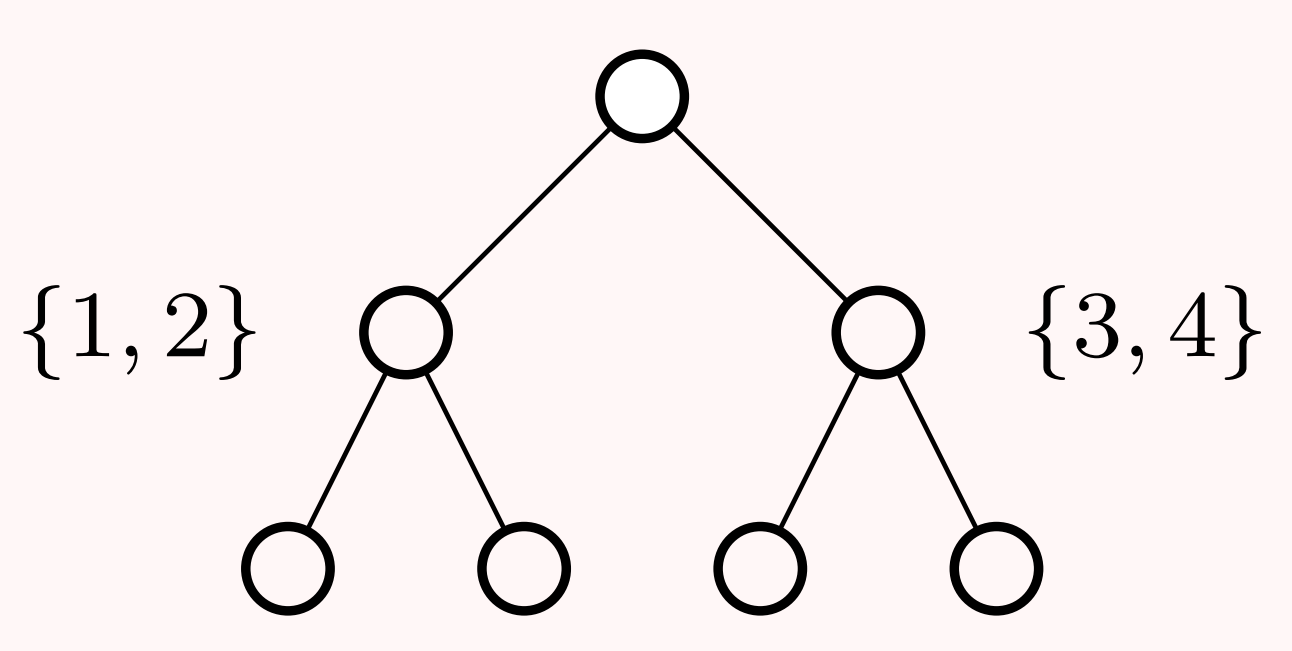

$\{1\} \quad\{2\} \quad\{3\} \quad\{4\}$

A dimension partition tree T over $D=\{1,2,3,4\}$

\section{Anthony Nouy ${ }^{1}$ \\ ${ }^{2}$ CEA/DAM/DIF, F-91297, Arpajon, France \\ Higher-order principal component analysis for tree-based formats}

The best approximation of $u$ by a function with $\alpha$-rank $r_{\alpha}$ is the truncated singular value decomposition:

$$
u_{r_{\alpha}}\left(x_{\alpha}, x_{\alpha^{c}}\right)=\sum_{k=1}^{r_{\alpha}} \sigma_{k} v_{k}^{\alpha}\left(x_{\alpha}\right) v_{k}^{\alpha^{c}}\left(x_{\alpha^{c}}\right)
$$

$v_{1}^{\alpha}, \ldots, v_{r_{\alpha}}^{\alpha}$ are the $r_{\alpha} \alpha$-principal components of $u$ and $\operatorname{span}\left\{v_{1}^{\alpha}, \ldots, v_{r_{\alpha}}^{\alpha}\right\}=U_{\alpha}$ is the $\alpha$-principal subspace of $u$.

\section{Extension to tree-based formats:}

From the leaves of the tree to the root, determine the subspaces $U_{\alpha}$ of principal components of $u_{\alpha}=\mathcal{P}_{V_{\alpha}} u$ for all $\alpha$, with $\mathcal{P}_{V_{\alpha}}$ the orthogonal projection onto $U_{\alpha} \otimes L_{\mu_{\alpha}}^{2}$ :

$$
\begin{aligned}
& \text { if } S(\alpha)=\emptyset, V_{\alpha} \text { is a given } \\
& \text { approximation space }
\end{aligned}
$$
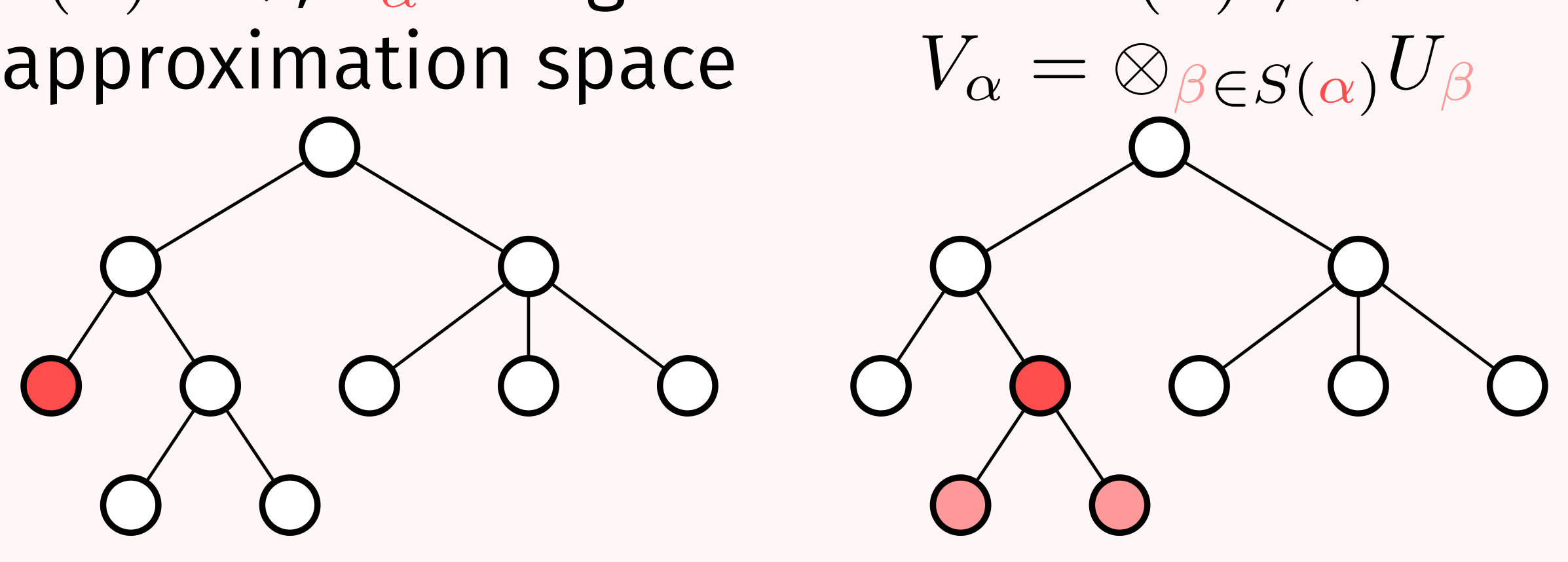

Finally project $\mathrm{u}$ onto the tensor space $\otimes_{\alpha \in S(D)} U_{\alpha}$, to obtain the approximation $u^{*}=\prod_{\alpha \in S(D)} \mathcal{P}_{U_{\alpha}} u$

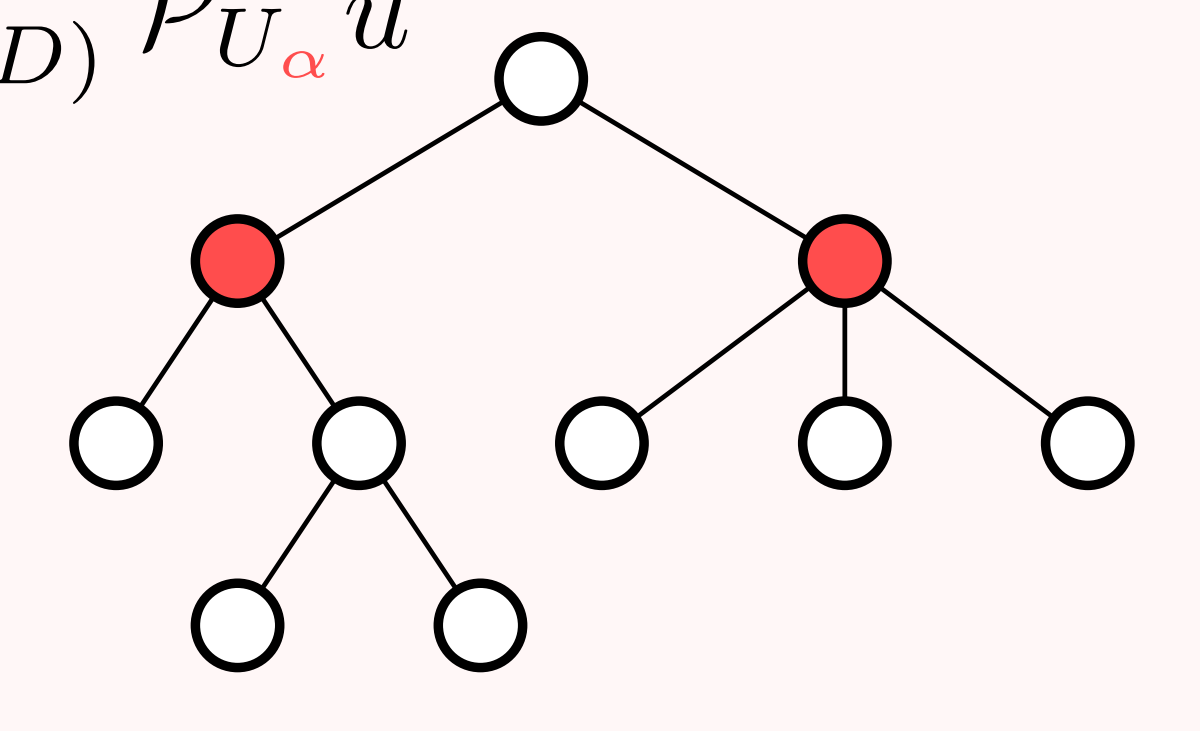

\section{Control of the error:}

- algorithm with prescribed rank:

$\left\|u-u^{*}\right\|^{2} \leq \# T \min _{v \in T_{r}^{T}}\|v-u\|^{2}+\sum_{\alpha \in \mathcal{L}(T)}\left\|u-\mathcal{P}_{V_{\alpha}} u\right\|^{2}$

- algorithm with prescribed tolerance:

if $\left\|u-\mathcal{P}_{V_{\alpha}} u\right\| \leq \epsilon / \sqrt{d}\|u\|$

and if $r_{\alpha}$ is chosen such that:

$\left\|u_{\alpha}-\mathcal{P}_{U_{\alpha}} u_{\alpha}\right\| \leq \epsilon / \sqrt{\# T}\left\|u_{\alpha}\right\|$

then $\left\|u-u^{*}\right\| \leq \epsilon\|u\|$

\section{Optimal Weighted Least-Squares}

PCA involves projections $P_{V} u$ with

$V=V_{\alpha}$ or $V=U_{\alpha}$ subspaces of $L_{\mu_{\alpha}}^{2}$.

For a function $f \in L_{\rho}^{2}$, replace the ideal orthogonal projection:

$$
P_{V} f=\arg \min _{v \in W}\|v-f\|_{L_{\rho}^{2}}
$$

by a weighted least-squares projection:

$$
P_{V}^{w} f=\arg \min _{v \in V} \frac{1}{n} \sum_{i=1}^{n} w^{i}\left|v\left(z^{i}\right)-f\left(z^{i}\right)\right|^{2}
$$

with $\left(z^{i}\right)_{i=1}^{n}$ i.i.d samples from the measure $d \rho_{w}$.

$\left\|P_{V}^{w} f-f\right\|_{L_{\rho}^{2}}$ should be close to the error of best approximation and $n$ as close as possible to $m$, the dimension of $V$.

\section{Optimal choice [1]:}

1. Sample $\left(z^{i}\right)_{i=1}^{n}$ from $d \rho_{w}=w^{-1} d \rho$ with $w^{-1}(z)=\frac{1}{m} \sum_{i=1}^{m} \varphi_{i}(z)$ where $\left(\varphi_{i}\right)_{i=1}^{m}$ is an orthonormal basis of $V$

2. Choose weigths $\left(w^{i}\right)_{i=1}^{n}=\left(w\left(z^{i}\right)\right)_{i=1}^{n}$

The condition $n \geq c(r) m \log (m)$ ensures the stability property:

$$
\operatorname{Pr}\left\{\|G-I\| \geq \frac{1}{2}\right\} \leq m^{-r}
$$

with $G$ the empirical grammian matrix of basis $\left(\varphi_{i}\right)_{i=1}^{m}$

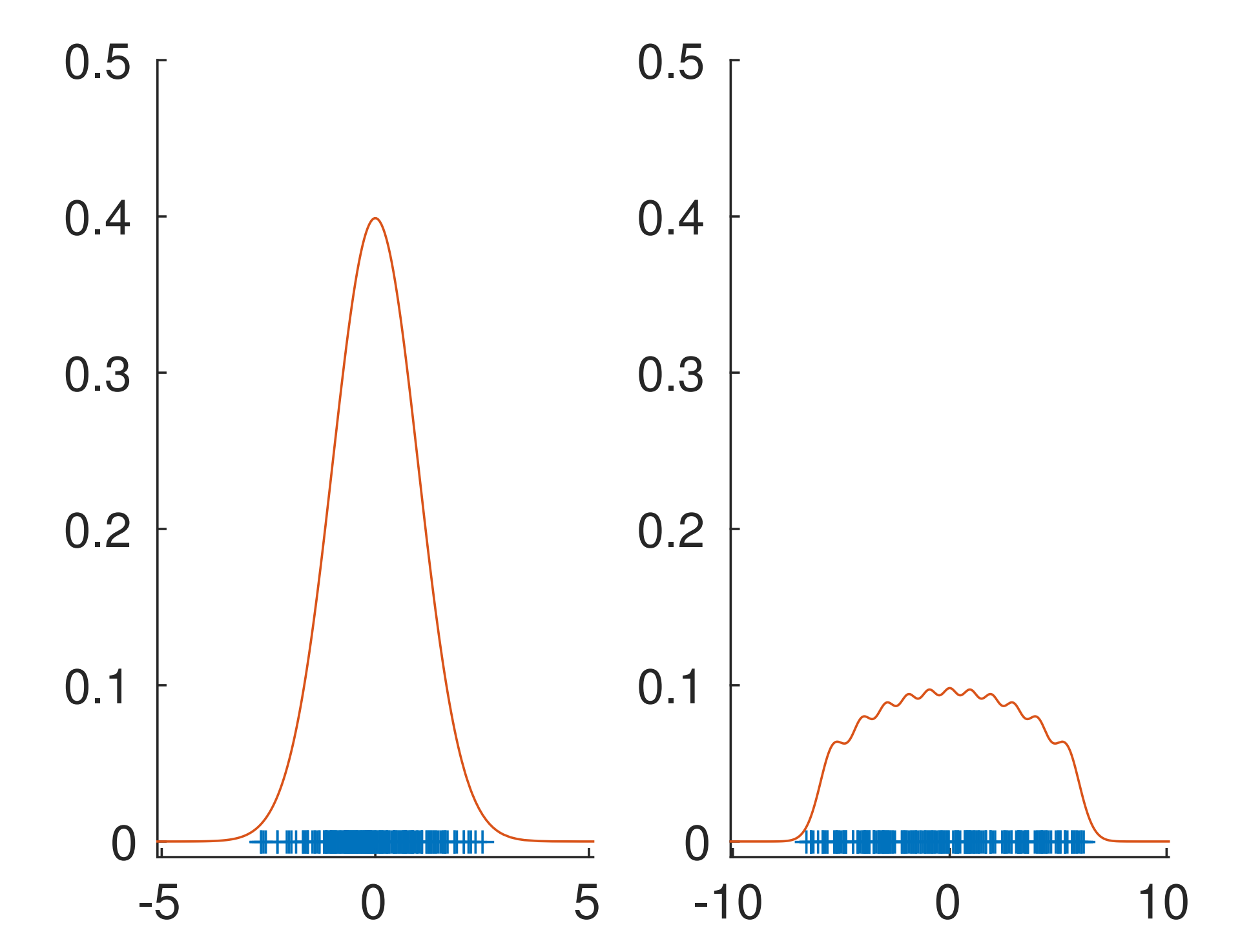

Left: Pdf and samples of the gaussian measure $\rho$, Right: Pdf and samples of the weighted measure $\rho_{w}$ with $m=11$

The sampling according to optimal measures exploits the tensor product structure of the functions involved.

\section{Conclusions}

Statistical estimation of principal components $U_{\alpha}$ In practice we determine $U_{\alpha}$ by solving: $\min _{\mathrm{m}\left(U_{\alpha}\right)=r_{\alpha}} \frac{1}{n_{\alpha}} \sum_{k=1}^{n_{\alpha}}\left\|u_{\alpha}\left(\cdot, x_{\alpha^{c}}^{k}\right)-P_{U_{\alpha}} u_{\alpha}\left(\cdot, x_{\alpha^{c}}^{k}\right)\right\|_{L_{\mu_{\alpha}}^{2}}$ $\left(x_{\alpha^{c}}^{k}\right)_{k=1}^{n_{\alpha}}$ are i.i.d. samples of the group of variables $x_{\alpha^{c}}$

$P_{U_{\alpha}}$ is the orthogonal projection onto $U_{\alpha}$.

\section{Numerical experiments $f(x)=\sin \left(\sum_{i=1}^{10} x_{i}\right)$}

We consider $\mathcal{X}=\mathbb{R}^{d}$, with the standard Gaussian measure, and a fixed $\alpha$-rank equal to 2 .

\begin{tabular}{c|ccc|ccc|}
$\begin{array}{c}\text { polynomial degree of } \\
\text { the approximation basis }\end{array}$ & \multicolumn{2}{|c}{ Approximation error in $\log _{10}$} & \multicolumn{2}{c}{ Number of samples } \\
WLS & SLS & Interpolation & WLS & SLS & Interpolation \\
\hline 5 & {$[-1.1,-0.9]$} & {$[-1,-0.1]$} & {$[-0.6,-0.4]$} & 1292 & 1292 & 188 \\
10 & {$[-3.6,-3.5]$} & {$[-3.4,-2.3]$} & {$[-4.3,-3.9]$} & 2212 & 2212 & 288 \\
20 & {$[-9.8,-9.5]$} & {$[-9.7,-7.5]$} & {$[-13.2,-10.8]$} & 4232 & 4232 & 488 \\
25 & {$[-13.3,-12.9]$} & {$[-8.8,-4.1]$} & {$[-13.1,-8.4]$} & 5292 & 5292 & 588 \\
40 & {$[-14.5,-14]$} & {$[-7.4,-2.5]$} & error & 8652 & 8652 & error
\end{tabular}

The optimal-weighted projection in the higher-order PCA algorithm increases the stability compared to a standard least-squares projection or interpolation. This stability is guaranteed with conditions on the number of samples.

\section{Ongoing works:}

- For a fixed rank, provide conditions on the number of samples $n_{\alpha}$ that garantee a quasi-optimality result in expectation or probability for the empirical PCA

- Provide fully adaptive algorithms (in samples, ranks) that achieve (in expectation or high probability) a prescribed precision

- Exploit sparsity of tensors

\section{References}

[1] A. Cohen and G. Migliorati. Optimal weighted least-squares methods. SMAI Journal of Computational Mathematics, 3:181-203, 2017.

[2] A. Nouy. Higher-order principal component analysis for the approximation of tensors in tree-based low rank formats. arXiv:1705.00880v1, 2017. 